\title{
Individual allergens as risk factors for asthma and bronchial hyperresponsiveness in Chinese children
}

\author{
G.W.K. Wong*, S.T. Li", D.S.C. Hui", T.F. Fok*, N.S. Zhong", Y.Z. Chen ${ }^{+}$, C.K.W. Lai ${ }^{\#}$
}

Individual allergens as risk factors for asthma and bronchial hyperresponsiveness in Chinese children. G.W.K. Wong, S.T. Li, D.S.C. Hui, T.F. Fok, N.S. Zhong, Y.Z. Chen, C.K.W. Lai. C ERS Journals Ltd 2002.

ABSTRACT: The role of allergen sensitization in the development of asthma in the Chinese is not clear. This study aims to determine the relationship of sensitization to individual allergens, and the development of asthma and bronchial hyperresponsiveness (BHR) in schoolchildren from three Chinese cities: Hong Kong, Beijing and Guangzhou.

Community-based random samples of 10-yr-old schoolchildren from three Chinese cities were recruited for study using the International Study of Asthma and Allergies in Childhood (ISAAC) Phase II protocol. Subjects were studied by parental questionnaires $(n=10,902)$, skin-prick tests $(n=3,479)$, and methacholine challenge tests $(n=608)$.

The prevalence rates of wheeze in the past 12 months (Hong Kong, 5.8\%; Beiiing, 3.8\%; Guangzhou, 3.4\%) and atopy (Hong Kong, 41.2\%; Beijing, 23.9\%; Guangzhou, 30.8\%) were highest in schoolchildren from Hong Kong. Multivariate logistic regression analyses revealed that sensitization to Dermatophagoides pteronyssinus (odds ratio $(\mathrm{OR})=4.48$; $95 \%$ confidence interval (CI): 3.02-6.66), cat $(2.59 ; 1.67-4.03)$, Dermatophagoides farinae $(2.41 ; 1.65-3.51)$ and mixed grass pollen $(2.85 ; 1.24-6.50)$ were significantly associated with current wheeze. Atopy, defined as having $\geqslant 1$ positive skin-prick tests, was not an independent risk factor for current wheeze in children from any of the three cities. Furthermore, atopy $(\mathrm{OR}=2.53 ; 95 \%$ CI: 1.07-5.97), sensitization to cat $(3.01 ; 1.39-6.52)$ and $D$. farinae $(3.67 ; 1.93-6.97)$ were significantly associated with BHR.

The authors confirmed that sensitization to house dust mite and cat was significantly associated with current wheeze and bronchial hyperresponsiveness in Chinese schoolchildren. However, the difference in the prevalence rate of atopic sensitization cannot explain the higher prevalence of childhood asthma in Hong Kong, when compared with those children from Beijing and Guangzhou.

Eur Respir J 2002; 19: 288-293.
Depts of *Paediatrics and "Medicine and Therapeutics, Prince of Wales Hospital, The Chinese University of Hong Kong, Shatin, Hong Kong Special Administrative Region, China, Guangzhou Institute of Respiratory Disease, Guangzhou, ${ }^{+}$Clinical and Education Centre for Asthma, Capital Institute of Paediatrics, Beijing, China.

Correspondence: C.K.W. Lai, Room 1403, Takshing House, 20 Des Voeux Road, Central, Hong Kong Special Administrative Region, China. Fax: 85225222188

E-mail: keilai@netvigator.com

Keywords: Allergen

asthma

atopy

bronchial hyperresponsiveness

children

Chinese

Received: March 272001

Accepted after revision September 17 2001

This study was supported by Research Grant Council Earmarked Grant 96/97 No. CUHK 232/96M.
Asthma is one of the most common chronic disorders in childhood. Although there is no gold standard for defining asthma, the diagnosis of asthma in clinical practice is made on the basis of combined information from history, physical examination, and respiratory function tests. Bronchial hyperresponsiveness (BHR) is the best objective marker of asthma and has been considered as one of the criteria for defining asthma epidemiologically $[1,2]$. As documented by the International Study of Asthma and Allergies in Childhood (ISAAC), the prevalence rates of asthma in schoolchildren vary widely across different regions of the world [3]. Furthermore, many epidemiological studies have consistently documented increasing prevalence of asthma and allergies in different countries [4-8]. The wide variation of asthma prevalence and the recent increasing trend within populations are unlikely to be explained by genetic factors. Environmental factors have been thought to be important in determining disease manifestations. Among the suspected environmental factors associated with asthma, allergen sensitization has been most widely implicated as one of the important risk factors [9].
The prevalence of asthma and allergic diseases in the Chinese population has been reported to be lower than that in the West [3]. Within the Chinese population, the prevalence rate of asthma in schoolchildren was found to be the highest in Hong Kong [3]. The 12-month prevalence rates of wheeze in 13-14-yr-old schoolchildren, as identified by the ISAAC written and video questionnaires, were $12.4 \%$ and $10.1 \%$, respectively in Hong Kong while the corresponding figures in mainland China were $4.2 \%$ and $2.0 \%$. Allergic sensitization could be one of the factors accounting for such differences between children from Hong Kong and mainland China. Hong Kong is a westernized city with a subtropical climate. Guangzhou is situated approximately $200 \mathrm{~km}$ north of Hong Kong and has a similar climate. Beijing, however, is in the temperate zone with relatively low humidity compared to the other two cities. The majority of published studies on the relationship of allergic sensitization and asthma were performed in Caucasians. A small comparative study of three populations of Chinese schoolchildren was conducted previously to assess the relationship of allergic 
sensitization and asthma [10]. The findings, however, were not validated with objective measures such as a bronchial challenge test. Only one previous study has utilized bronchial challenge tests in Chinese children, and BHR was shown to be associated with atopy [11]. However, the relative importance of different allergens has not been assessed in the Chinese population.

In this study, the relationship of sensitization to individual allergens and the development of asthmatic symptoms and BHR were evaluated, in schoolchildren from three Chinese cities.

\section{Subjects and methods}

The details of subject selection and methods used in this study have been reported elsewhere [12]. In brief, schoolchildren aged 10 yrs from Hong Kong, Beijing and Guangzhou were recruited into the study using primary schools as sampling units. Each subject was given an ISAAC Phase II questionnaire [13] to be completed by the parents or guardians. "Current" symptoms referred to symptoms in the past 12 months. Random subgroups of at least 1,000 children from each city were recruited for skin-prick tests after parental consent was obtained, as described previously [12]. The sensitivity to eight common aeroallergens was assessed: Dermatophagoides pteronyssinus, Dermatophagoides farinae, cat, Alternaria tenuis, mixed tree pollen, mixed grass pollen, cockroach, and mixed moulds. Standardized allergen extracts and control solutions were obtained from ALK (Horsholm, Denmark). Histamine $\left(10 \mathrm{mg} \cdot \mathrm{mL}^{-1}\right)$ and diluent were used as positive and negative controls. A drop of each allergen extract was brought into the skin of the volar side of the left forearm using ALK lancets. After $15 \mathrm{~min}$, the weal reaction was measured as the mean of the longest diameter and the length of the perpendicular line through its middle. A weal size $\geqslant 3 \mathrm{~mm}$ after subtraction of the negative control was considered positive. Subjects with $\geqslant 1$ positive reactions were considered atopic. To ensure the quality of the skin-prick tests, the procedure was performed by trained technicians and strict adherance to the ISAAC Phase II protocol was observed.

All children with current wheeze identified by the written questionnaire were invited for bronchial challenge test. Equal numbers of control subjects were randomly recruited from all students who reported no symptom of wheeze in the past year. The test was carried out according to the protocol reported by YAN et al. [14]. BHR is defined as having a provocation dose of methacholine causing a $20 \%$ fall in forced expiratory volume in one second from baseline (PD20) of $\leqslant 7.8 \mu \mathrm{mol}$ methacholine. The study protocol was approved by the Ethics Committee of the participating institutions. Informed parental consent was obtained from parents or guardians before the studies were carried out.

All data were entered into a computer twice, by two independent investigators. Data were categorized and analysed statistically [15]. Multivariate logistic regression analysis with adjustment for age and sex was used to test the associations of sensitivity of each allergen, as well as atopy and the dependent variables (current wheeze and $\mathrm{BHR}$ ). Initially, univariate analyses (i.e. analysis performed without adjusting for any of the covariates) were performed. Only allergens found to be statistically significant in the univariate analyses were then tested in a combined analysis to reveal the independent risk factors. The results of the multivariate logistic regression analyses are reported. Results are presented as odds ratios (OR) and 95\% confidence interval (CI). A p-value $<0.05$ was considered to be statistically significant. To compare the effects of atopy and allergen sensitization in the three cities, multivariate logistic regression analyses were performed separately for children from each city. The data of all children were then pooled together in the final analyses with adjustment for the place of residence.

\section{Results}

The prevalence rates of asthma symptoms and atopic sensitization in the three study populations, as revealed by the written questionnaires and skin-prick tests are summarized in table 1. Asthma symptoms and atopy were significantly more common in schoolchildren from Hong Kong when compared with those from Beijing and Guangzhou. Similarly, sensitivity to $D$. pteronyssinus and $D$. farinae was significantly more common in Hong Kong than in Beijing or Guangzhou. The prevalence of sensitivity to mixed moulds, however, was highest in Beijing, and it was significantly higher than those in Hong Kong or Guangzhou. A total of 459 subjects reported to have wheeze in the past 12 months. There were 179 from Hong Kong, 159 from Beijing and 121 from Guangzhou. A total of 285 of these subjects (62\%) gave consent to undergo the bronchial challenge test. Subjects $(n=323)$ without current wheeze were also recruited from three cities for bronchial challenge test. Therefore, a total of 608 subjects (Hong Kong, 238; Beijing, 193; Guangzhou, 177) underwent bronchial challenge test. Among them, $515(85 \%)$ also gave consent to have skin-prick tests.

Multivariate logistic regression analyses with adjustment for age and sex revealed that sensitivity to $D$. pteronyssinus, $D$. farinae, cat, mixed grass pollen, and mixed moulds were risk factors significantly associated with current wheeze. Table 2 shows the relationship of individual allergens as risk factors for current wheeze in the three cities. Among the allergens tested, sensitivity to $D$. pteronyssinus and $D$. farinae were the most common risk factors associated with current wheeze in the three groups of schoolchildren. Sensitivity to mixed moulds was most common in Beijing (3.2\%), and it was found to be a risk factor for current wheeze in schoolchildren from Beijing only. Atopy was not associated with current wheeze in any of the three groups of children.

Table 3 shows the relationship of individual allergens as risk factors for BHR. When subjects from all three cities were analysed as one combined group, atopy, sensitivity to $D$. farinae and cat were found to be risk factors for BHR. Sensitivity to 
Table 1. - Prevalence of asthmatic symptoms and atopic sensitization in Chinese schoolchildren by study area

\begin{tabular}{lccc}
\hline & Hong Kong & Beijing & Guangzhou \\
\hline Written questionnaire $\mathrm{n}$ & 3110 & 4227 & 3565 \\
Symptoms in last 12 months & & & \\
Wheeze & $5.8(5.0-6.7)$ & $3.8(3.3-4.4)$ & $3.4(2.8-4.1)$ \\
Exercise-induced wheeze & $7.7(6.8-8.6)$ & $4.5(3.9-5.2)$ & $3.1(2.5-3.7)$ \\
Speech-limiting wheezing & $1.8(1.3-2.3)$ & $0.5(0.3-0.7)$ & $0.4(0.3-0.7)$ \\
Current asthma & $3.3(2.7-4.1)$ & $2.3(1.9-2.8)$ & $2.1(1.7-2.6)$ \\
Skin-prick test n & 1341 & 1044 & 1094 \\
Atopy ( 1 positive skin test) & $41.2(38.6-43.8)$ & $23.9(21.4-26.6)$ & $30.8(28.1-33.7)$ \\
Dermatophagoides pteronyssinus & $34.1(31.5-36.7)$ & $7.5(5.9-9.3)$ & $20.0(17.7-22.5)$ \\
Dermatophagoides farinae & $25.9(23.6-28.3)$ & $5.7(4.3-7.2)$ & $17.9(15.7-20.3)$ \\
Cat & $3.7(2.8-4.9)$ & $5.6(4.3-7.1)$ & $4.3(3.2-5.7)$ \\
Cockroach & $11.5(9.8-13.3)$ & $13.3(11.3-15.6)$ & $17.3(15.1-19.7)$ \\
Mixed grass pollen & $0.7(0.4-1.4)$ & $1.2(0.7-2.1)$ & $1.1(0.6-1.9)$ \\
Alternaria tenuis & $0.4(0.1-0.9)$ & $1.5(0.9-2.5)$ & $0.9(0.4-1.7)$ \\
Mixed moulds & $0.4(0.2-1.0)$ & $3.2(2.2-4.4)$ & $1.2(0.6-2.0)$ \\
Mixed tree pollen & $0.1(0.0-0.4)$ & $1.0(0.5-1.8)$ & $0.5(0.2-1.2)$ \\
Subjects $\mathrm{n}$ & & 193 & 177 \\
Bronchial challenge test & 238 & 91 & 82 \\
Current wheeze & 112 & 102 & 95 \\
No current wheeze & 126 & & \\
\hline
\end{tabular}

Data are presented as $\mathrm{n} \%(95 \%$ confidence interval) unless otherwise stated.

D. pteronyssinus was found to be of borderline significance in predicting $\mathrm{BHR}(\mathrm{p}=0.07$; $\mathrm{OR}=2.04$; 95\% CI: 0.944 .43 ). Although sensitivity to $D$. farinae confers the highest risk $(\mathrm{OR}=3.67)$ for $\mathrm{BHR}$, the differences between the ORs of this, atopy and cat were not significantly different. For the individual cities, sensitivity to $D$. pteronyssinus and $D$. farinae were the most common factors associated with BHR.

\section{Discussion}

Epidemiological studies have documented a progressive increase in the prevalence and morbidity of asthma, which is now one of the most common chronic disorders in childhood. The prevalence rates of asthma and allergic disorders in Asians have been found to be lower when compared with those in the West [16]. This research group has also documented an increasing trend of asthma in schoolchildren from Hong Kong [3]. There is limited data available regarding the role of sensitization to allergens in the development of asthma and bronchial hyperreactivity in Chinese children [11].

The current study is the first to evaluate the relative importance of sensitization to individual allergens for the development of asthma and BHR in Chinese schoolchildren. Using a standardized protocol, the authors confirmed that sensitization to D. pteronyssi$n u s$, D. farinae and cat, were risk factors associated with current wheeze and BHR in Chinese schoolchildren. The authors have validated previously that

Table 2. - Individual allergens as risk factors for current wheeze in Chinese children

\begin{tabular}{lllrl}
\hline Allergen sensitivity & \multicolumn{1}{c}{ Hong Kong } & \multicolumn{1}{c}{ Beijing } & Guangzhou & Total \\
\hline Atopy & $1.54(0.65-3.62)$ & $0.66(0.20-2.22)$ & $2.94(0.92-9.32)$ & $1.29(0.74-2.24)$ \\
Dermatophagoides pteronyssinus & $3.03(1.82-5.06)^{*}$ & $2.28(0.60-8.66)$ & $11.65(5.44-24.94)^{*}$ & $4.48(3.02-6.66)^{*}$ \\
Cat & $1.46(0.69-3.08)$ & $2.11(0.68-6.57)$ & $6.79(3.06-15.02)^{*}$ & $2.59(1.67-4.03)^{*}$ \\
Dermatophagoides farinae & $1.67(1.02-2.74)^{*}$ & $8.53(3.74-19.42)^{*}$ & $3.73(1.87-7.45)^{*}$ & $2.41(1.65-3.51)^{*}$ \\
Mixed grass pollen & $5.26(1.45-19.16)^{*}$ & $0.34(0.03-3.76)$ & $5.32(0.87-32.53)$ & $2.85(1.24-6.50)^{*}$ \\
Mixed moulds & $0.64(0.06-7.14)$ & $3.30(1.10-9.87)^{*}$ & $0.85(0.21-3.52)$ & $1.30(0.60-2.80)$ \\
\hline
\end{tabular}

Data are represented as odds ratios $(95 \%$ confidence interval). $*$ : $\mathrm{p}<0.05$.

Table 3. - Individual allergens as risk factors for bronchial hyperresponsiveness in Chinese children

\begin{tabular}{llccc}
\hline Allergen sensitivity & \multicolumn{1}{c}{ Hong Kong } & Beijing & Guangzhou & Total \\
\hline Atopy & $4.57(1.90-11.00)^{*}$ & $2.29(0.35-15.24)$ & $2.20(0.42-11.45)$ & $2.53(1.07-5.97)^{*}$ \\
Dermatophagoides pteronyssinus & $1.19(0.37-3.88)$ & $13.18(3.08-56.48)^{*}$ & $4.76(1.61-14.08)^{*}$ & $2.04(0.94-4.43)^{\#}$ \\
Cat & $1.64(0.48-5.60)$ & $6.18(1.17-32.59)^{*}$ & $3.34(0.89-15.51)$ & $3.01(1.39-6.52)^{*}$ \\
Dermatophagoides farinae & $2.56(1.12-5.84)^{*}$ & $0.66(0.05-8.46)$ & $9.52(3.28-27.66)^{*}$ & $3.67(1.93-6.97)^{*}$ \\
\hline
\end{tabular}

Data are represented as odds ratios $\left(95 \%\right.$ confidence interval). ${ }^{*}: \mathrm{p}<0.05 ;{ }^{*}: \mathrm{p}=0.07$. 
the Chinese version of the ISAAC written questionnaire was effective in predicting BHR in secondary schoolchildren in Hong Kong [17]. The current study used the same protocol of bronchial challenge test. When all of the subjects recruited for BHR testing from the three cities $(n=608)$ were combined, a positive response to the question on current wheeze had a sensitivity of 0.82 and a specificity of 0.71 for predicting BHR. A potential source of bias in this study was that only $62 \%$ of the children with current wheeze gave consent for BHR testing. The impact of such bias, however, is likely to be small because the prevalence of symptoms of eczema and rhinoconjunctivitis, family history of atopy, as well as the frequency of wheezing attacks in the subgroup who underwent BHR testing, were virtually identical to those of the whole group of children with current wheeze (data not shown).

House dust mite exposure and sensitization in early childhood have been found to be associated with subsequent development of asthma in Caucasians [18-20]. Studies of asthmatic children have also revealed an association between asthma symptoms, BHR and atopy [21, 22]. Furthermore, exposure to mite allergen in asthmatic children has been found to be associated with the risk of repeated hospital admissions [23]. vON Mutius et al. [24] found a higher prevalence of asthma in schoolchildren in West Germany compared with those in East Germany and suggested that the difference in prevalence rates might be explained by sensitization to aeroallergens. There has been only one small comparative study of Chinese children investigating the role of allergen sensitization in the manifestation of asthma [10]. This study recruited Chinese schoolchildren aged $12-18$ yrs from Hong Kong, Malaysia and San Bu, China. A subsample underwent skin-prick testing to determine the relationship of allergen sensitization and asthma. Asthma and allergic disorder were 2-6-times higher in schoolchildren from Hong Kong when compared with the other two groups of Chinese children. Within each study population, allergen sensitization was a significant factor associated with asthma. The lack of objective assessment of airway response in this study was a major limitation. Furthermore, the prevalence of atopy in Malaysia was high $(64 \%)$ and yet the prevalence of asthma was low (1.9\%). In addition to allergic sensitization, other factors associated with the "westernized" environment of Hong Kong may also be important for the development of asthma in schoolchildren from Hong Kong [25]. A recent review emphasized that the proportion of cases of childhood asthma attributable to atopy, varied from only $25-63 \%$ in cross-sectional studies. Indeed, the present study shows that atopy per se is not an independent risk factor for current wheeze. Thus, research efforts should also be directed at other possible aetiological factors for the development of asthma [26].

Cockroach allergy and exposure to high levels of this allergen have also been found to be associated with increased morbidity in asthmatic children in America [27, 28]. However, a recent study of German schoolchildren did not find a relationship between cockroach sensitization and asthma symptoms [29].
The results shown in the present study are in agreement with the German study. Although the prevalence of cockroach sensitization is common in all three cities, such sensitization was neither associated with current wheeze nor BHR, in the subjects studied. Further studies are required to define clearly the role of cockroach allergy in the manifestation of asthma in different populations. In addition, atopy, defined as having $\geqslant 1$ positive skin tests, was associated with current wheeze in the univariate analyses in the subjects studied (data not shown). However, atopy was not independently associated with current wheeze in any of the three groups of children. This finding suggests that asthma symptoms have a strong relationship with sensitization to particular aeroallergens, but not with the nonspecific response of just being atopic alone [26, 30-35]. Furthermore, there are other possible confounders that may influence the results, such as family history of atopy and smoking in the household. When these two factors were included in the statistical model of the analyses, the significant associations remained unchanged (data not shown). However, studies with larger sample sizes are required to clearly define the possible interactions of these factors, upon the associations of allergen sensitization with asthma or BHR.

Finally, it was found that sensitization to house dust mite was significantly more common in schoolchildren from Hong Kong than those from Beijing or Guangzhou. Sensitization to house dust mite was confirmed to be significantly associated with current wheeze and BHR in the studied subjects; therefore, the high prevalence rate of childhood asthma in Hong Kong may be due partly to the high prevalence rate of sensitization to house dust mite. However, the difference in sensitization rate cannot be explained by the difference in allergen exposure because previously the authors have shown that domestic indoor levels of aeroallergens in Hong Kong and Guangzhou were similar [36]. Furthermore, despite a significantly higher rate of sensitization to house dust mite in Guangzhou when compared with that of Beijing, the prevalence of asthma symptoms were similar in Beijing and Guangzhou. Therefore, other factors such as those implicated in the hygiene hypothesis, breast-feeding and dietary factors, most likely also influence the susceptibility in the development of allergen sensitization and asthma.

In conclusion, the authors have evaluated three populations of Chinese schoolchildren using standardized protocol of skin-prick test and bronchial challenge test. This is the first report of the relative importance of sensitization to individual allergens for asthma symptoms and bronchial hyperresponsiveness in Chinese children. The authors have confirmed that sensitization to house dust mite and cat is significantly associated with current wheeze and bronchial hyperresponsiveness in Chinese schoolchildren. However, the difference in the prevalence rates of atopic sensitization among the three cities cannot explain the higher prevalence of childhood asthma in Hong Kong when compared with children from Mainland China. Further studies are necessary to determine the factors which influence the susceptibility in the 
development of allergen sensitization in Chinese children.

\begin{abstract}
Acknowledgements. The authors wish to thank M. Tong, A. Chan, C. Chan and K.K.Wong for their technical support, and the children, parents and staff of the schools for their participation in the survey.
\end{abstract}

\section{References}

1. Sterk PJ, Fabbri LM, Quanjer PH, et al. Airway responsiveness: standardized challenge testing with pharmacological, physical and sensitizing stimuli in adults. Eur Respir J 1993; 6: 53-83.

2. Pekkanen J, Pearce N. Defining asthma in epidemiological studies. Eur Respir J 1999; 14: 951-957.

3. Leung R, Wong GWK, Lau J, et al. Prevalence of asthma and allergy in Hong Kong schoolchildren: an ISAAC study. Eur Respir J 1997; 10: 354-360.

4. Hsieh KH, Shen JJ. Prevalence of childhood asthma in Taipei, Taiwan and other Asian Pacific countries. J Asthma 1988; 25: 73-82.

5. Aberg N, Hesselmar B, Aberg B, Eriksson B. Increase of asthma, allergic rhinitis and eczema in Swedish schoolchildren between 1979 and 1991. Clin Exp Allergy 1995; 25: 815-819.

6. Burr ML, Butland BK, King S, Vaughau-Williams E. Changes in asthma prevalence: two surveys 15 years apart. Arch Dis Child 1989; 64: 1118-1125.

7. Shaw RA, Crane J, O'Donnell TV, Porteous LE, Coleman ED. Increasing asthma prevalence in a rural New Zealand adolescent population 1975-89. Arch Dis Child 1990; 65: 1319-1323.

8. Venn A, Lewis S, Cooper M, Hill J, Britton J. Increasing prevalence of wheeze and asthma in Nottingham primary schoolchildren 1988-1995. Eur Respir J 1998; 11: 1324-1328.

9. Warner JO. Bronchial hyperresponsiveness, atopy, airway inflammation, and asthma. Pediatr Allergy Immunol 1998; 9: 56-60.

10. Leung R, Ho P, Lam CWK, Lai CKW. Sensitization to inhaled allergens as a risk factor for asthma and allergic diseases in Chinese population. $J$ Allergy Clin Immunol 1997; 99: 594-599.

11. Zhong NS, Chen RC, O-yang M, Wu JY, Fu WX, Shi LJ. Bronchial hyperresponsiveness in young students of southern China: relationship to respiratory symptoms, diagnosed asthma, and risk factors. Thorax 1990; 45: 860-865.

12. Wong GWK, Hui DSC, Chan HH, et al. Prevalence of respiratory and atopic disorders in Chinese schoolchildren. Clin Exp Allergy 2001; 31: 1225-1231.

13. The International Study of Asthma and Allergies in Childhood (ISAAC) Steering Committee. Phase II Modules of the International Study of Asthma and Allergies in Childhood (ISAAC). Munster, 1998.

14. Yan K, Salome C, Woolcock AJ. Rapid method for measurement of bronchial responsiveness. Thorax 1983; 38: 760-765.

15. SPSS for Windows Release 10.0. Maija J Norusis/ SPSS Inc. 1999.

16. The International Study of Asthma and Allergies in Childhood (ISAAC) Steering Committee. Worldwide variations in the prevalence of asthma symptoms: the
International Study of Asthma and Allergies in Childhood (ISAAC). Eur Respir J 1998; 12: 315335.

17. Lai CKW, Chan JKW, Chan A, et al. Comparison of the ISAAC video questionnaire (AVQ 3.0) with the ISAAC written questionnaire for estimating asthma associated bronchial hyperreactivity. Clin Exp Allergy 1997; 27: 540-545.

18. Sporik R, Holgate S, Platts-Mills TAE, Cogswell JJ. Exposure to house dust mite (der p1) and the development of asthma in childhood. $N$ Eng $\mathrm{J} \mathrm{Med}$ 1990; 323: 502-507.

19. Peat JK, Salome CM, Woolcock AJ. Longitudinal changes in atopy during a 4 year period: relation to bronchial hyperresponsiveness and respiratory symptoms in a population sample of Australian schoolchildren. J Allergy Clin Immunol 1990; 85: 65-74.

20. Kuehr J, Frischer T, Meinert R, et al. Sensitization to mite allergens is a risk factor for early and late onset of asthma and for persistence of asthmatic signs in children. J Allergy Clin Immunol 1995; 95: 655662.

21. Sears MR, Herbison GP, Holdaway MD, Hewitt CJ, Flannery EM, Silva PA. The relative risks of sensitivity to grass pollen, house dust mite and cat dander in the development of childhood asthma. Clin Exp Allergy 1989; 19: 419-424.

22. Burrows B, Sears MR, Flannery EM, Herbison GP, Holdaway MD. Relations of bronchial responsiveness to allergy skin test reactivity, lung function, respiratory symptoms, and diagnoses in thirteen-year-old New Zealand children. J Allergy Clin Immunol 1995; 95: 548-556.

23. Sporik R, Platts-Mills TAE, Cogswell JJ. Exposure to house dust mite allergen of children admitted to hospital with asthma. Clin Exp Allergy 1993; 27: 740746.

24. von Mutius E, Martinez FD, Fritzsch C, Nicolai T, Roell G, Thiemann H-H. Prevalence of asthma and atopy in two areas of West and East Germany. Am J Respir Crit Care Med 1994; 149: 358-364.

25. Seaton A, Godden DJ, Brown K. Increase in asthma: a more toxic environment or a more susceptible population. Thorax 1994; 49: 171-174.

26. Pearce N, Pekkanen J, Beasley R. How much asthma is really attributable to atopy? Thorax 1999; 54: 268 272.

27. Rosenstreich DL, Eggleston P, Kattan M, et al. The role of cockroach allergy and exposure to cockroach allergen in causing morbidity among inner-city children with asthma. N Engl J Med 1997; 336: 13561363.

28. Sporik R, Squillace SP, Ingram JM, Rakes G, Honsinger RW, Platts-Mills TA. Mite, cat, and cockroach exposure, allergen sensitization and asthma in children: a case-control study of three schools. Thorax 1999; 54: 675-680.

29. Hirsch T, Stappenbeck C, Neumeister V, et al. Exposure and allergic sensitization to cockroach allergen in East Germany. Clin Exp Allergy 2000; 30: 529-537.

30. Sears MR, Burrows B, Flannery EM, Herbison GP, Hewitt CJ, Holdaway MD. Relation between airway responsiveness and serum $\mathrm{IgE}$ in children with asthma and in apparently normal children. $N$ Engl $\mathrm{J} \mathrm{Med}$ 1991; 325: 1067-1071.

31. Custovic A, Taggart SCO, Francis HC, Chapman 
MD, Woodcock A. Exposure to house dust mite allergens and the clinical activity of asthma. $J$ Allergy Clin Immunol 1996; 98: 64-72.

32. Peat JK, Tovey E, Toelle BG, et al. House dust mite allergens as a major risk factor for childhood asthma in Australia. Am J Allergy Clin Immunol 1996; 153: 141-146.

33. Squillace SP, Sporik RB, Rakes G, et al. Sensitization to dust mites as a dominant risk factor for asthma among adolescents living in Central Virginia. Am J Respir Crit Care Med 1997; 156: 1760-1764.

34. Chinn S, Burney P, Sunyer J, Jarvis D, Luczynska C.
Sensitization to individual allergens and bronchial responsiveness in the ECRHS. Eur Respir $J$ 1999; 14: 876-884.

35. Sears MR, Herbison GP, Holdaway MD, Hewitt CJ, Flannery EM, Silva PA. The relative risks of sensitivity to grass pollen, house dust mite and cat dander in the development of childhood asthma. Clin Exp Allergy 1989; 19: 419-424.

36. Lai CKW, Wong GWK, Chan IH, Wong HY, Leung $\mathrm{R}$, Zhong NS. Domestic indoor areo-allergen levels in southern China-Hong Kong and Guangzhou. Eur Respir J 1998; 12: 441s. 